\title{
Stability of functional equation obtained through a fixed-point alternative in intuitionistic fuzzy normed spaces
}

\author{
SA Mohiuddine* and Mohammed A Alghamdi
}

"Correspondence:

mohiuddine@gmail.com Department of Mathematics,

Faculty of Science, King Abdulaziz University, P.O. Box 80203, Jeddah,

21589, Saudi Arabia

\begin{abstract}
In this paper, we determine the stability of a generalized Hyers-Ulam-Rassias-type theorem concerning the additive functional equation $2 f\left(\frac{x+y+z}{2}\right)=f(x)+f(y)+f(z)$ in the framework of intuitionistic fuzzy normed spaces through the fixed-point alternative. Further, we prove some stability results of an additive functional equation in this setup through the direct method.

MSC: 39B52; 39B82; 46540

Keywords: $t$-norm; $t$-conorm; additive functional equation; intuitionistic fuzzy normed space; fixed point
\end{abstract}

\section{Introduction}

The study of the stability problem of functional equations originated from a question of S.M. Ulam [30] concerning the stability of group homomorphisms.

Let $(G, *)$ be a group and $\left(G^{\prime}, \circ, d\right)$ be a metric group with the metric $d(\cdot, \cdot)$. Given $\epsilon>$ 0 , does there exist a $\delta(\epsilon)>0$ such that if a mapping $h: G \rightarrow G^{\prime}$ satisfies the inequality $d(h(x * y), h(x) \circ h(y))<\delta$ for all $x, y \in G$, then there exists a homomorphism $H: G \rightarrow G^{\prime}$ with $d(h(x), H(x))<\epsilon$ for all $x \in G$ ?

If the answer is affirmative, we would say the equation of homomorphism $H(x * y)=$ $H(x) \circ H(y)$ is stable. The concept of stability for a functional equation arises when we replace the functional equation by an inequality which acts as a perturbation of the equation. Hyers [5] gave a first affirmative partial answer to the question of Ulam for Banach spaces. Hyers theorem was generalized by Aoki [3] for additive mappings and by Rassias [26] for linear mappings by considering an unbounded Cauchy difference $\|f(x+y)-f(x)-f(y)\| \leq$ $\epsilon\left(\|x\|^{p}+\|y\|^{p}\right)$ for all $\epsilon>0$ and $p \in[0,1)$. Following the same approach as Rassias, Gajda [7] gave an affirmative solution of this problem for $p>1$ and also proved that it is possible to solve the Rassias-type theorem for $p=1$. A further generalization was obtained by Găvruta [8], who replaced $\epsilon\left(\|x\|^{p}+\|y\|^{p}\right)$ by a general control function $\varphi(x, y)$. The paper of Rassias has significantly influenced the development of what we now call the Hyers-Ulam-Rassias stability of functional equations. Since then, several stability problems for various functional equations have been investigated in [1, 2, 6, 9-12, 27, 32]. Quite recently, the stability problem for the Pexiderized quadratic functional equation, Jensen functional equation, cubic functional equation, functional equations associated with inner product spaces, and a mixed type additive-cubic functional equation were considered in $[15,17,21,29]$, and 
[31], respectively, in the intuitionistic fuzzy normed spaces; while the idea of intuitionistic fuzzy normed space was introduced in [28], and further studied in [18-20, 22-24, 34] to deal with some summability problems.

In 2003, Radu [25] proposed that the fixed-point alternative method is very useful for obtaining the solution of the Ulam problem and obtained the stability of the Cauchy functional equation in Banach spaces through the fixed-point method. Since then, several stability problems of this concept have been established by various authors, e.g., $[13,14,16$, 33] and references therein.

The aim of this paper is to present a relationship between three various disciplines: the theory of fuzzy spaces, the theory of functional equations, and fixed-point theory. We determine the stability of the additive functional equation

$$
2 f\left(\frac{x+y+z}{2}\right)=f(x)+f(y)+f(z)
$$

in the setting of intuitionistic fuzzy normed spaces by using the fixed-point alternative theorem. Also, we investigate the stability of this functional equation through the direct method.

\section{Definitions, notations and preliminary results}

In this section, we recall some notations, basic definitions, and preliminary results used in this paper.

A binary operation $*:[0,1] \times[0,1] \rightarrow[0,1]$ is said to be a continuous $t$-norm if it satisfies the following conditions:

(a) $*$ is associative and commutative,

(b) $*$ is continuous,

(c) $a * 1=a$ for all $a \in[0,1]$,

(d) $a * b \leq c * d$ whenever $a \leq c$ and $b \leq d$ for each $a, b, c, d \in[0,1]$.

A binary operation $\diamond:[0,1] \times[0,1] \rightarrow[0,1]$ is said to be a continuous $t$-conorm if it satisfies the following conditions:

$\left(\mathrm{a}^{\prime}\right) \diamond$ is associative and commutative,

$\left(\mathrm{b}^{\prime}\right) \diamond$ is continuous,

(c') $a \diamond 0=a$ for all $a \in[0,1]$,

(d') $a \diamond b \leq c \diamond d$ whenever $a \leq c$ and $b \leq d$ for each $a, b, c, d \in[0,1]$.

Using the notions of continuous $t$-norm and $t$-conorm, Saadati and Park [28] have recently introduced the concepts of intuitionistic fuzzy normed space and defined convergence and Cauchy sequences in this setting as follows.

Definition 2.1 The five-tuple $(X, \mu, v, *, \diamond)$ is said to be an intuitionistic fuzzy normed spaces (for short, IFN-Spaces) if $X$ is a vector space, $*$ is a continuous $t$-norm, $\diamond$ is a continuous $t$-conorm, and $\mu, v$ are fuzzy sets on $X \times(0, \infty)$ satisfying the following conditions. For every $x, y \in X$ and $s, t>0$

(i) $\mu(x, t)+v(x, t) \leq 1$,

(ii) $\mu(x, t)>0$,

(iii) $\mu(x, t)=1$ if and only if $x=0$, 
(iv) $\mu(\alpha x, t)=\mu\left(x, \frac{t}{|\alpha|}\right)$ for each $\alpha \neq 0$,

(v) $\mu(x, t) * \mu(y, s) \leq \mu(x+y, t+s)$,

(vi) $\mu(x, \cdot):(0, \infty) \rightarrow[0,1]$ is continuous,

(vii) $\lim _{t \rightarrow \infty} \mu(x, t)=1$ and $\lim _{t \rightarrow 0} \mu(x, t)=0$,

(viii) $v(x, t)<1$,

(ix) $v(x, t)=0$ if and only if $x=0$,

(x) $v(\alpha x, t)=v\left(x, \frac{t}{|\alpha|}\right)$ for each $\alpha \neq 0$,

(xi) $v(x, t) \diamond v(y, s) \geq v(x+y, t+s)$,

(xii) $v(x, \cdot):(0, \infty) \rightarrow[0,1]$ is continuous,

(xiii) $\lim _{t \rightarrow \infty} v(x, t)=0$ and $\lim _{t \rightarrow 0} v(x, t)=1$.

In this case, $(\mu, v)$ is called an intuitionistic fuzzy norm. For simplicity in notation, we denote the intuitionistic fuzzy normed spaces by $(X, \mu, v)$ instead of $(X, \mu, v, *, \diamond)$. For example, let $(X,\|\cdot\|)$ be a normed space, and let $a * b=a b$ and $a \diamond b=\min \{a+b, 1\}$ for all $a, b \in[0,1]$. For all $x \in X$ and every $t>0$, consider

$$
\mu(x, t):=\frac{t}{t+\|x\|} \quad \text { and } \quad v(x, t):=\frac{\|x\|}{t+\|x\|} .
$$

Then $(X, \mu, v)$ is an intuitionistic fuzzy normed space.

Definition 2.2 Let $(X, \mu, v)$ be an intuitionistic fuzzy normed space. Then a sequence $x=\left(x_{k}\right)$ is said to be

(i) convergent to $L \in X$ with respect to the intuitionistic fuzzy norm $(\mu, v)$ if, for every $\epsilon>0$ and $t>0$, there exists $k_{0} \in \mathbb{N}$ such that $\mu\left(x_{k}-L, t\right)>1-\epsilon$ and $v\left(x_{k}-L, t\right)<\epsilon$ for all $k \geq k_{0}$. In this case, we write $(\mu, v)-\lim x_{k}=L$ or $x_{k} \stackrel{(\mu, v)}{\longrightarrow} L$ as $k \rightarrow \infty$.

(ii) Cauchy sequence with respect to the intuitionistic fuzzy norm $(\mu, v)$ if, for every $\epsilon>0$ and $t>0$, there exists $k_{0} \in \mathbb{N}$ such that $\mu\left(x_{k}-x_{\ell}, t\right)>1-\epsilon$ and $v\left(x_{k}-x_{\ell}, t\right)<\epsilon$ for all $k, \ell \geq k_{0}$. IFN-space $(X, \mu, \nu)$ is said to be complete if every Cauchy sequence in $(X, \mu, v)$ is convergent in IFN-space. In this case, $(X, \mu, v)$ is called intuitionistic fuzzy Banach space.

Remark 2.3 Let $(X,\|\cdot\|)$ be a real normed linear space,

$$
\mu:=\frac{t}{t+\|x\|} \quad \text { and } \quad v:=\frac{\|x\|}{t+\|x\|}
$$

for all $x \in X$ and $t>0$. Then $x_{n} \stackrel{\|\cdot\|}{\longrightarrow} x$ if and only if $x_{n} \stackrel{(\mu, v)}{\longrightarrow} x$.

Recall the following results related to the concept of fixed point.

Theorem 2.4 (Banach's contraction principle) Let $(X, d)$ be a complete generalized metric space and consider a mapping $J: X \rightarrow X$ be a strictly contractive mapping, that is,

$$
d(J x, J y) \leq L d(x, y), \quad \forall x, y \in X
$$

for some (Lipschitz constant) $L<1$. Then

(i) The mapping $J$ has one and only one fixed point $x^{*}=J\left(x^{*}\right)$; 
(ii) The fixed-point $x^{*}$ is globally attractive, that is,

$$
\lim _{n \rightarrow \infty} J^{n} x=x^{*}
$$

for any starting point $x \in X$;

(iii) One has the following estimation inequalities for all $x \in X$ and $n \geq 0$ :

$$
\begin{aligned}
& d\left(J^{n} x, x^{*}\right) \leq L^{n} d\left(x, x^{*}\right), \\
& d\left(J^{n} x, x^{*}\right) \leq \frac{1}{1-L} d\left(J^{n} x, J^{n+1} x\right), \\
& d\left(x, x^{*}\right) \leq \frac{1}{1-L} d(x, J x) .
\end{aligned}
$$

Theorem 2.5 (The alternative of fixed point [4]) Suppose we are given a complete generalized metric space $(X, d)$ and a strictly contractive mapping $J: X \rightarrow X$, with Lipschitz constant $L$. Then, for each given element $x \in X$, either

$$
d\left(J^{n} x, J^{n+1} x\right)=+\infty, \quad \forall n \geq 0
$$

or

$$
d\left(J^{n} x, J^{n+1} x\right)<+\infty, \quad \forall n \geq n 。
$$

for some natural number $n_{0}$. Moreover, if the second alternative holds then

(i) The sequence $\left(J^{n} x\right)$ is convergent to a fixed point $y^{*}$ of $J$;

(ii) $y^{\prime \prime}$ is the unique fixed point of $J$ in the set $Y=\left\{y \in X, d\left(J^{n_{\circ}} x, y\right)<+\infty\right\}$

(iii) $d\left(y, y^{\prime \prime}\right) \leq \frac{1}{1-L} d(y, J y), y \in Y$.

\section{Stability of the additive functional equation through the fixed-point alternative}

Using the fixed point alternative, here we can prove the stability of the Hyers-UlamRassias-type theorem in IFN-spaces. First, we prove the following lemma which will be used in our main result.

Lemma 3.1 Let $X$ be a linear space, $(Y, \mu, v)$ be an IFN-space and $\varphi: X \times X \times X \rightarrow[0, \infty)$ be a function. Consider a set $G=\{g: X \rightarrow Y\}$ and define

$$
\begin{aligned}
d_{s}(g, h)= & \inf \left\{\gamma \in \mathbb{R}^{+}: \mu(g(x)-h(x), \gamma t) \geq \frac{t}{t+\varphi(x, 2 x, x)}\right. \text { and } \\
& \left.\nu(g(x)-h(x), \gamma t) \leq \frac{\varphi(x, 2 x, x)}{t+\varphi(x, 2 x, x)}\right\}
\end{aligned}
$$

for all $g, h \in G, x \in X$ and $t>0$. Then $d_{s}$ is a complete generalized metric on $G$.

Proof Let $g, h, k \in G, d_{s}(g, h)<\gamma_{1}$ and $d_{s}(h, k)<\gamma_{2}$. Then, for all $x \in X$ and $t>0$, we have

$$
\mu\left(g(x)-h(x), \gamma_{1} t\right) \geq \frac{t}{t+\varphi(x, 2 x, x)}, \quad \mu\left(h(x)-k(x), \gamma_{2} t\right) \geq \frac{t}{t+\varphi(x, 2 x, x)} ;
$$


and

$$
v\left(g(x)-h(x), \gamma_{1} t\right) \leq \frac{\varphi(x, 2 x, x)}{t+\varphi(x, 2 x, x)}, \quad v\left(h(x)-k(x), \gamma_{2} t\right) \leq \frac{\varphi(x, 2 x, x)}{t+\varphi(x, 2 x, x)}
$$

Therefore,

$$
\mu\left(g(x)-k(x),\left(\gamma_{1}+\gamma_{2}\right) t\right) \geq \mu\left(g(x)-h(x), \gamma_{1} t\right) * \mu\left(h(x)-k(x), \gamma_{2} t\right) \geq \frac{t}{t+\varphi(x, 2 x, x)}
$$

and

$$
v\left(g(x)-k(x),\left(\gamma_{1}+\gamma_{2}\right) t\right) \leq v\left(g(x)-h(x), \gamma_{1} t\right) \diamond v\left(h(x)-k(x), \gamma_{2} t\right) \leq \frac{\varphi(x, 2 x, x)}{t+\varphi(x, 2 x, x)}
$$

for each $x \in X$ and $t>0$. Thus, $d_{s}(g, k) \leq \gamma_{1}+\gamma_{2}$, which is a triangle inequality for $d_{s}$. The rest of the conditions follow directly from the definition.

Theorem 3.2 Let $X$ be a linear space and $f$ be a mapping from $X$ to an intuitionistic fuzzy Banach space $(Y, \mu, v)$. Suppose that $\varphi: X \times X \times X \rightarrow[0, \infty)$ is a function such that

$$
\left.\begin{array}{l}
\mu\left(2 f\left(\frac{x+y+z}{2}\right)-f(x)-f(y)-f(z), t\right) \geq \frac{t}{t+\varphi(x, y, z)} \quad \text { and } \\
\nu\left(2 f\left(\frac{x+y+z}{2}\right)-f(x)-f(y)-f(z), t\right) \leq \frac{\varphi(x, y, z)}{t+\varphi(x, y, z)},
\end{array}\right\}
$$

for all $x, y, z \in X$ and $t>0$. If $\varphi(x, y, z) \leq \frac{\alpha}{2} \varphi(2 x, 2 y, 2 z)$ holds for some real number $\alpha$ with $\alpha<1$ then there exists a unique additive mapping $T: X \rightarrow Y$ such that $T(x)=$ $(\mu, v)-\lim _{n \rightarrow \infty} 2^{n} f\left(\frac{x}{2^{n}}\right)$

$$
\begin{aligned}
& \mu(f(x)-T(x), t) \geq \frac{(2-2 \alpha) t}{(2-2 \alpha) t+\alpha \varphi(x, 2 x, x)} \quad \text { and } \\
& \nu(f(x)-T(x), t) \leq \frac{\alpha \varphi(x, 2 x, x)}{(2-2 \alpha) t+\alpha \varphi(x, 2 x, x)},
\end{aligned}
$$

for all $x \in X$ and $t>0$.

Proof Putting $y=2 x$ and $z=x$ in (3.2). Then for $x \in X$ and $t>0$

$$
\begin{aligned}
& \mu(f(2 x)-2 f(x), t) \geq \frac{t}{t+\varphi(x, 2 x, x)} \quad \text { and } \\
& \nu(f(2 x)-2 f(x), t) \leq \frac{\varphi(x, 2 x, x)}{t+\varphi(x, 2 x, x)} .
\end{aligned}
$$

Replacing $x$ by $x / 2$, we get

$$
\begin{aligned}
& \mu\left(2 f\left(\frac{x}{2}\right)-f(x), t\right) \geq \frac{t}{t+\varphi\left(\frac{x}{2}, x, \frac{x}{2}\right)} \quad \text { and } \\
& \mu\left(2 f\left(\frac{x}{2}\right)-f(x), t\right) \geq \frac{\varphi\left(\frac{x}{2}, x, \frac{x}{2}\right)}{t+\varphi\left(\frac{x}{2}, x, \frac{x}{2}\right)} .
\end{aligned}
$$


Consider the set $G=\{g: X \rightarrow Y\}$ and the mapping $d$ defined on $G \times G$ by

$$
\begin{aligned}
d_{s}(g, h)= & \inf \left\{\gamma \in \mathbb{R}^{+}: \mu(g(x)-h(x), \gamma t) \geq \frac{t}{t+\varphi(x, 2 x, x)}\right. \text { and } \\
& \left.v(g(x)-h(x), \gamma t) \leq \frac{\varphi(x, 2 x, x)}{t+\varphi(x, 2 x, x)}\right\}
\end{aligned}
$$

for all $x \in X$ and $t>0$. It is known that $d_{s}(g, h)$ is a complete generalized metric on $G$ by Lemma 3.1. Now we consider the linear mapping $J: G \rightarrow G$ such that $g(x)=2 g\left(\frac{x}{2}\right)$ for all $x \in X$. Let $g, h \in G$ be such that $d_{s}(g, h)=\xi$. Then, for all $x \in X$ and $t>0$, we have

$$
\mu(g(x)-h(x), \xi t) \geq \frac{t}{t+\varphi(x, 2 x, x)} \quad \text { and } \quad v(g(x)-h(x), \xi t) \leq \frac{\varphi(x, 2 x, x)}{t+\varphi(x, 2 x, x)} .
$$

Using the hypothesis of the function $\varphi$ and a mapping $J$, we obtain

$$
\mu(J g(x)-J h(x), \alpha \xi t)=\mu\left(g\left(\frac{x}{2}\right)-h\left(\frac{x}{2}\right), \frac{\alpha \xi t}{2}\right) \geq \frac{\frac{\alpha t}{2}}{\frac{\alpha t}{2}+\varphi\left(\frac{x}{2}, x, \frac{x}{2}\right)} \geq \frac{\frac{\alpha t}{2}}{\frac{\alpha t}{2}+\frac{\alpha}{2} \varphi(x, 2 x, x)},
$$

this implies

$$
\mu(J g(x)-J h(x), \alpha \xi t) \geq \frac{t}{t+\varphi(x, 2 x, x)}
$$

and similarly

$$
v(J g(x)-J h(x), \alpha \xi t)=v\left(g\left(\frac{x}{2}\right)-h\left(\frac{x}{2}\right), \frac{\alpha \xi t}{2}\right) \geq \frac{\varphi\left(\frac{x}{2}, x, \frac{x}{2}\right)}{\frac{\alpha t}{2}+\varphi\left(\frac{x}{2}, x, \frac{x}{2}\right)} \geq \frac{\varphi(x, 2 x, x)}{t+\varphi(x, 2 x, x)},
$$

for all $x \in X$ and $t>0$. From above, we conclude that $d_{s}(g, h)=\xi$ implies $d_{s}(g g, J h) \leq \alpha \xi$. Hence,

$$
d_{s}(J g, J h) \leq \alpha d_{s}(g, h)
$$

for all $g, h \in G$. Using the hypothesis of the function $\varphi$ and from (3.4), we have

$$
\begin{aligned}
& \mu\left(2 f\left(\frac{x}{2}\right)-f(x), t\right) \geq \frac{\frac{2 t}{\alpha}}{\frac{2 t}{\alpha}+\varphi(x, 2 x, x)} \quad \text { and } \\
& \nu\left(2 f\left(\frac{x}{2}\right)-f(x), t\right) \geq \frac{\varphi(x, 2 x, x)}{\frac{2 t}{\alpha}+\varphi(x, 2 x, x)},
\end{aligned}
$$

for all $x \in X, t>0$ and $\alpha<1$. Replacing $t$ by $\frac{\alpha t}{2}$ in (3.5), we get

$$
\begin{aligned}
& \mu\left(f(x)-2 f\left(\frac{x}{2}\right), \frac{\alpha t}{2}\right) \geq \frac{t}{t+\varphi(x, 2 x, x)} \quad \text { and } \\
& \nu\left(f(x)-2 f\left(\frac{x}{2}\right), \frac{\alpha t}{2}\right) \geq \frac{\varphi(x, 2 x, x)}{t+\varphi(x, 2 x, x)}
\end{aligned}
$$


for all $x \in X, t>0$ and $\alpha<1$. It follows that

$$
d_{s}(f, J f) \leq \frac{\alpha}{2}
$$

Using the fixed-point alternative we deduce the existence of a fixed point of $J$, that is, the existence of a mapping $T: X \rightarrow Y$ such that

$$
T\left(\frac{x}{2}\right)=\frac{T(x)}{2}
$$

for all $x \in X$. The mapping $T$ is a unique fixed point of $J$ in the set $E=\left\{h \in G: d_{s}(g, h)<\infty\right\}$. It follows that $T$ is the unique fixed point of $J$ with the property that there exists $c \in(0, \infty)$ such that

$$
\mu(g(x)-h(x), c t) \geq \frac{t}{t+\varphi(x, 2 x, x)} \quad \text { and } \quad v(g(x)-h(x), c t) \leq \frac{\varphi(x, 2 x, x)}{t+\varphi(x, 2 x, x)},
$$

for all $x \in X$ and $t>0$. Moreover, we have $d\left(J^{n} f, T\right) \rightarrow 0$ as $n \rightarrow \infty$ which implies

$$
(\mu, v)-\lim _{n \rightarrow \infty} 2^{n} f\left(\frac{x}{2^{n}}\right)=T(x)
$$

for all $x \in X$. Also $d_{s}(f, T) \leq \frac{1}{1-\alpha} d_{s}(f, J f)$ implies $d_{s}(f, T) \leq \frac{\alpha}{2-2 \alpha}$. This means that (3.3) holds. For all $x, y, z \in X$ and $t>0$, write

$$
\begin{aligned}
& \mu\left(2 T\left(\frac{x+y+z}{2}\right)-T(x)-T(y)-T(z), t\right) \\
& \geq \mu\left(2 T\left(\frac{x+y+z}{2}\right)-2^{n+1} f\left(\frac{x+y+z}{2^{n+1}}\right), \frac{t}{5}\right) * \mu\left(2^{n} f\left(\frac{x}{2^{n}}\right)-T(x), \frac{t}{5}\right) \\
& \quad * \mu\left(2^{n} f\left(\frac{y}{2^{n}}\right)-T(y), \frac{t}{5}\right) * \mu\left(2^{n} f\left(\frac{z}{2^{n}}\right)-T(z), \frac{t}{5}\right) \\
& \quad * \mu\left(2^{n+1} f\left(\frac{x+y+z}{2^{n+1}}\right)-2^{n} f\left(\frac{x}{2^{n}}\right)-2^{n} f\left(\frac{y}{2^{n}}\right)-2^{n} f\left(\frac{z}{2^{n}}\right), \frac{t}{5}\right) .
\end{aligned}
$$

Letting $n \rightarrow \infty$ in (3.6) and using (3.2), we get

$$
\mu\left(2 T\left(\frac{x+y+z}{2}\right)-T(x)-T(y)-T(z), t\right)=1 .
$$

Similarly, we obtain

$$
v\left(2 T\left(\frac{x+y+z}{2}\right)-T(x)-T(y)-T(z), t\right)=0,
$$

for all $x, y, z \in X$ and $t>0$. Thus, the mapping $T$ satisfies (1.1) and so it is additive.

Corollary 3.3 Let $X$ be a normed linear space and $(Y, \mu, v)$ be an intuitionistic fuzzy Banach space. Let $\theta$ be a positive real number and $r$ is a real number with $r>1$. If a mapping 
$f: X \rightarrow Y$ satisfies the conditions

$$
\begin{aligned}
& \mu\left(2 f\left(\frac{x+y+z}{2}\right)-f(x)-f(y)-f(z), t\right) \geq \frac{t}{t+\theta\left(\|x\|^{r}+\|y\|^{r}+\|z\|^{r}\right)} \text { and } \\
& \nu\left(2 f\left(\frac{x+y+z}{2}\right)-f(x)-f(y)-f(z), t\right) \leq \frac{\theta\left(\|x\|^{r}+\|y\|^{r}+\|z\|^{r}\right)}{t+\theta\left(\|x\|^{r}+\|y\|^{r}+\|z\|^{r}\right)},
\end{aligned}
$$

for all $x, y, z \in X$ and $t>0$, then there exists a unique additive mapping $T: X \rightarrow Y$ such that $T(x)=(\mu, v)-\lim _{n \rightarrow \infty} 2^{n} f\left(\frac{x}{2^{n}}\right)$,

$$
\begin{aligned}
& \mu(f(x)-T(x), t) \geq \frac{\left(2^{r}-1\right) t}{\left(2^{r}-1\right) t+\left(2^{r-1}+1\right) \theta\|x\|^{r}} \text { and } \\
& v(f(x)-T(x), t) \leq \frac{\left(2^{r-1}+1\right) \theta\|x\|^{r}}{\left(2^{r}-1\right) t+\left(2^{r-1}+1\right) \theta\|x\|^{r}},
\end{aligned}
$$

for all $x \in X$ and $t>0$.

Proof Taking $\varphi(x, y, z)=\theta\left(\|x\|^{r}+\|y\|^{r}+\|z\|^{r}\right)$ in Theorem 3.2, for all $x, y, z \in X$, and choosing $\alpha=2^{-r}$, we get the desired result.

Theorem 3.4 Let $X$ be a linear space and $\varphi: X \times X \times X \rightarrow[0, \infty)$ be a function such that there exists $\alpha<1$ with $\varphi(2 x, 2 y, 2 z) \leq 2 \alpha \varphi(x, y, z)$ for all $x, y, z \in X$ and $t>0$. Suppose $f$ is a mapping from $X$ to an intuitionistic fuzzy Banach space $(Y, \mu, v)$ satisfying (3.2). Then there exists a unique additive mapping $T: X \rightarrow Y$ such that $T(x)=(\mu, v)-\lim _{n \rightarrow \infty} \frac{f\left(2^{n} x\right)}{2^{n}}$,

$$
\begin{aligned}
& \mu(f(x)-T(x), t) \geq \frac{(2-2 \alpha) t}{(2-2 \alpha) t+\varphi(x, 2 x, x)} \quad \text { and } \\
& \nu(f(x)-T(x), t) \leq \frac{\varphi(x, 2 x, x)}{(2-2 \alpha) t+\varphi(x, 2 x, x)},
\end{aligned}
$$

for all $x \in X$ and $t>0$.

Proof Consider a complete generalized metric space $\left(G, d_{s}\right)$ same as in the proof of Theorem 3.2. We define a linear mapping $J: G \rightarrow G$ such that

$$
g(x)=\frac{g(2 x)}{2}
$$

for all $x \in X$. Indeed, for given $g$ and $h$ in $G, d_{s}(g, h)=\xi$. Then

$$
\mu(g(x)-h(x), \xi t) \geq \frac{t}{t+\varphi(x, 2 x, x)} \quad \text { and } \quad v(g(x)-h(x), \xi t) \leq \frac{\varphi(x, 2 x, x)}{t+\varphi(x, 2 x, x)},
$$

for all $x \in X$ and $t>0$. By the given hypothesis and using (3.8), we have

$$
\mu(J g(x)-J h(x), \alpha \xi t)=\mu(g(2 x)-h(2 x), 2 \alpha \xi t) \geq \frac{2 \alpha t}{2 \alpha t+\varphi(2 x, 4 x, 2 x)} \geq \frac{t}{t+\varphi(x, 2 x, x)}
$$

and

$$
v(J g(x)-J h(x), \alpha \xi t)=v(g(2 x)-h(2 x), 2 \alpha \xi t) \leq \frac{\varphi(2 x, 4 x, 2 x)}{2 \alpha t+\varphi(2 x, 4 x, 2 x)} \geq \frac{\varphi(x, 2 x, x)}{t+\varphi(x, 2 x, x)},
$$


for all $x \in X$ and $t>0$. This means that $d_{s}(g g, J h) \leq \alpha \xi$. Thus, $d_{s}(g g, J h) \leq \alpha d_{s}(g, h)$ for all $g$ and $h$ in G. It follows from (3.2) that

$$
\begin{aligned}
& \mu\left(\frac{f(2 x)}{2}-f(x), \frac{t}{2}\right) \geq \frac{t}{t+\varphi(x, 2 x, x)} \quad \text { and } \\
& \nu\left(\frac{f(2 x)}{2}-f(x), \frac{t}{2}\right) \geq \frac{\varphi(x, 2 x, x)}{t+\varphi(x, 2 x, x)},
\end{aligned}
$$

for all $x \in X$ and $t>0$. From the definition of complete generalized metric space, we have $d_{s}(f, J f) \leq \frac{1}{2}$. Using the fixed-point alternative, we deduce the existence of a fixed point of $J$, that is, the existence of a mapping $T: X \rightarrow Y$ such that $2 T(x)=T(2 x)$ for all $x \in X$. Moreover, we have $d\left(J^{n} f, T\right) \rightarrow 0$ which implies

$$
(\mu, v)-\lim _{n \rightarrow \infty} \frac{f\left(2^{n} x\right)}{2^{n}}=T(x)
$$

for all $x \in X$. Also $d_{s}(f, T) \leq \frac{1}{1-\alpha} d_{s}(f, J f)$ implies $d_{s}(f, T) \leq \frac{1}{2-2 \alpha}$. The rest of the proof can be done by the same way as in Theorem 3.2.

Corollary 3.5 Let $X$ be a normed linear space and $(Y, \mu, v)$ be an intuitionistic fuzzy Banach space. Let $\theta$ be a positive real number and $r$ is a real number with $0<r<\frac{1}{3}$. If a mapping $f: X \rightarrow Y$ satisfies the conditions,

$$
\begin{aligned}
& \mu\left(2 f\left(\frac{x+y+z}{2}\right)-f(x)-f(y)-f(z), t\right) \geq \frac{t}{t+\theta\left(\|x\|^{r}+\|y\|^{r}+\|z\|^{r}\right)} \text { and } \\
& \nu\left(2 f\left(\frac{x+y+z}{2}\right)-f(x)-f(y)-f(z), t\right) \leq \frac{\theta\left(\|x\|^{r}+\|y\|^{r}+\|z\|^{r}\right)}{t+\theta\left(\|x\|^{r}+\|y\|^{r}+\|z\|^{r}\right)}
\end{aligned}
$$

for all $x, y, z \in X$ and $t>0$, then there exists a unique additive mapping $T: X \rightarrow Y$ such that $T(x)=(\mu, v)-\lim _{n \rightarrow \infty} \frac{f\left(2^{n} x\right)}{2^{n}}$,

$$
\begin{aligned}
& \mu(f(x)-T(x), t) \geq \frac{\left(2^{3 r}-1\right) t}{\left(2^{3 r}-1\right) t+\left(2^{3 r}+2^{4 r-1}\right) \theta\|x\|^{r}} \text { and } \\
& \nu(f(x)-T(x), t) \leq \frac{\left(2^{3 r}+2^{4 r-1}\right) \theta\|x\|^{r}}{\left(2^{3 r}-1\right) t+\left(2^{3 r}+2^{4 r-1}\right) \theta\|x\|^{r}}
\end{aligned}
$$

for all $x \in X$ and $t>0$.

Proof Taking $\varphi(x, y, z)=\theta\left(\|x\|^{r}+\|y\|^{r}+\|z\|^{r}\right)$ in Theorem 3.4, for all $x, y, z \in X$, and choosing $\alpha=2^{-3 r}$, we get the desired result.

\section{Stability of the additive functional equation through the direct method}

In this section, we deal with the stability results concerning the additive functional equation via direct method in intuitionistic fuzzy normed spaces. 
Theorem 4.1 Let $X$ be a linear space and $\left(Z, \mu^{\prime}, v^{\prime}\right)$ be an IFN-space. Suppose that $\varphi$ : $X \times X \times X \rightarrow Z$ is a function such that for some real number $\alpha$ with $0<|\alpha|<1 / 2$

$$
\begin{aligned}
& \mu^{\prime}\left(\varphi\left(\frac{x}{2}, \frac{y}{2}, \frac{z}{2}\right), t\right) \geq \mu^{\prime}\left(\varphi(x, y, z), \frac{t}{|\alpha|}\right) \text { and } \\
& v^{\prime}\left(\varphi\left(\frac{x}{2}, \frac{y}{2}, \frac{z}{2}\right), t\right) \leq v^{\prime}\left(\varphi(x, y, z), \frac{t}{|\alpha|}\right),
\end{aligned}
$$

for all $x, y, z \in X$ and $t>0$. Let $f$ be a mapping from $X$ to an intuitionistic fuzzy Banach $\operatorname{space}(Y, \mu, v)$ such that

$$
\left.\begin{array}{l}
\mu\left(2 f\left(\frac{x+y+z}{2}\right)-f(x)-f(y)-f(z), t\right) \geq \mu^{\prime}(\varphi(x, y, z), t) \quad \text { and } \\
\nu\left(2 f\left(\frac{x+y+z}{2}\right)-f(x)-f(y)-f(z), t\right) \leq v^{\prime}(\varphi(x, y, z), t),
\end{array}\right\}
$$

for all $x, y, z \in X$ and $t>0$. Then there exists a unique additive mapping $T: X \rightarrow Y$ such that

$$
\left.\begin{array}{l}
\mu(f(x)-T(x), t) \geq \mu^{\prime}\left(\varphi(x, 2 x, x), \frac{(1-2|\alpha|) t}{|\alpha|}\right) \quad \text { and } \\
v(f(x)-T(x), t) \leq v^{\prime}\left(\varphi(x, 2 x, x), \frac{(1-2|\alpha|) t}{|\alpha|}\right),
\end{array}\right\}
$$

for all $x \in X$ and $t>0$.

Proof Put $y=2 x$ and $z=x$ in (4.2). Then for all $x \in X$ and $t>0$

$$
\begin{aligned}
& \mu(f(2 x)-2 f(x), t) \geq \mu^{\prime}(\varphi(x, 2 x, x), t) \quad \text { and } \\
& v(f(2 x)-2 f(x), t) \leq v^{\prime}(\varphi(x, 2 x, x), t) .
\end{aligned}
$$

Replacing $x$ by $\frac{x}{2^{j+1}}$ in (4.4) and using (4.1), we obtain

$$
\mu\left(2^{j+1} f\left(\frac{x}{2^{j+1}}\right)-2^{j} f\left(\frac{x}{2^{j}}\right), 2^{j} t\right) \geq \mu^{\prime}\left(\varphi\left(\frac{x}{2^{j+1}}, \frac{x}{2^{j}}, \frac{x}{2^{j+1}}\right), t\right) \geq \mu^{\prime}\left(\varphi(x, y, z), \frac{t}{|\alpha|^{j+1}}\right)
$$

and

$$
v\left(2^{j+1} f\left(\frac{x}{2^{j+1}}\right)-2^{j} f\left(\frac{x}{2^{j}}\right), 2^{j} t\right) \leq v^{\prime}\left(\varphi\left(\frac{x}{2^{j+1}}, \frac{x}{2^{j}}, \frac{x}{2^{j+1}}\right), t\right) \leq v^{\prime}\left(\varphi(x, y, z), \frac{t}{|\alpha|^{j+1}}\right),
$$

for all $x \in X, t>0$ and an integer $j \geq 0$. By replacing $t=|\alpha|^{j+1} t$, we get

$$
\left.\begin{array}{l}
\mu\left(2^{j+1} f\left(\frac{x}{2^{j+1}}\right)-2^{j} f\left(\frac{x}{2^{j}}\right), 2^{j}|\alpha|^{j+1} t\right) \geq \mu^{\prime}(\varphi(x, y, z), t) \quad \text { and } \\
\nu\left(2^{j+1} f\left(\frac{x}{2^{j+1}}\right)-2^{j} f\left(\frac{x}{2^{j}}\right), 2^{j}|\alpha|^{j+1} t\right) \leq v^{\prime}(\varphi(x, y, z), t) .
\end{array}\right\}
$$


It follows from

$$
2^{n} f\left(\frac{x}{2^{n}}\right)-f(x)=\sum_{j=0}^{n-1}\left(2^{j+1} f\left(\frac{x}{2^{j+1}}\right)-2^{j} f\left(\frac{x}{2^{j}}\right)\right)
$$

and (4.5) that

$$
\begin{aligned}
\mu\left(2^{n} f\left(\frac{x}{2^{n}}\right)-f(x), \sum_{j=0}^{n-1} 2^{j}|\alpha|^{j+1} t\right) & \geq \prod_{j=0}^{n-1} \mu\left(2^{j+1} f\left(\frac{x}{2^{j+1}}\right)-2^{j} f\left(\frac{x}{2^{j}}\right), 2^{j}|\alpha|^{j+1} t\right) \\
& \geq \mu^{\prime}(\varphi(x, 2 x, x), t) \text { and } \\
v\left(2^{n} f\left(\frac{x}{2^{n}}\right)-f(x), \sum_{j=0}^{n-1} 2^{j}|\alpha|^{j+1} t\right) & \leq \coprod_{j=0}^{n-1} v\left(2^{j+1} f\left(\frac{x}{2^{j+1}}\right)-2^{j} f\left(\frac{x}{2^{j}}\right), 2^{j}|\alpha|^{j+1} t\right) \\
& \leq v^{\prime}(\varphi(x, 2 x, x), t),
\end{aligned}
$$

for all $x \in X, t>0$ and $n>0$, where $\prod_{i=1}^{n} a_{i}=a_{1} * a_{2} * \cdots * a_{n}$ and $\prod_{i=1}^{n} a_{i}=a_{1} \diamond a_{2} \diamond \cdots \diamond a_{n}$. Replacing $x$ by $\frac{x}{2^{p}}$ in the last inequalities, we have

$$
\begin{aligned}
& \mu\left(2^{n+p} f\left(\frac{x}{2^{n+p}}\right)-2^{p} f\left(\frac{x}{2^{p}}\right), \sum_{j=0}^{n-1} 2^{j+p}|\alpha|^{j+1} t\right) \\
& \geq \mu^{\prime}\left(\varphi\left(\frac{x}{2^{p}}, \frac{2 x}{2^{p}}, \frac{x}{2^{p}}\right), t\right) \geq \mu^{\prime}\left(\varphi(x, 2 x, x), \frac{t}{|\alpha|^{p}}\right) \text { and } \\
& \nu\left(2^{n+p} f\left(\frac{x}{2^{n+p}}\right)-2^{p} f\left(\frac{x}{2^{p}}\right), \sum_{j=0}^{n-1} 2^{j+p}|\alpha|^{j+1} t\right) \\
& \quad \leq v^{\prime}\left(\varphi\left(\frac{x}{2^{p}}, \frac{2 x}{2^{p}}, \frac{x}{2^{p}}\right), t\right) \leq v^{\prime}\left(\varphi(x, 2 x, x), \frac{t}{|\alpha|^{p}}\right),
\end{aligned}
$$

whence

$$
\begin{aligned}
& \mu\left(2^{n+p} f\left(\frac{x}{2^{n+p}}\right)-2^{p} f\left(\frac{x}{2^{p}}\right), \sum_{j=0}^{n-1} 2^{j+p}|\alpha|^{j+p+1} t\right) \geq \mu^{\prime}(\varphi(x, 2 x, x), t) \quad \text { and } \\
& \nu\left(2^{n+p} f\left(\frac{x}{2^{n+p}}\right)-2^{p} f\left(\frac{x}{2^{p}}\right), \sum_{j=0}^{n-1} 2^{j+p}|\alpha|^{j+p+1} t\right) \leq v^{\prime}(\varphi(x, 2 x, x), t),
\end{aligned}
$$

for all $x \in X, t>0, n>0$ and $p \geq 0$. Hence,

$$
\left.\begin{array}{l}
\mu\left(2^{n+p} f\left(\frac{x}{2^{n+p}}\right)-2^{p} f\left(\frac{x}{2^{p}}\right), t\right) \geq \mu^{\prime}\left(\varphi(x, 2 x, x), \frac{t}{\sum_{j=p}^{n+p-1} 2^{j}|\alpha|^{j+1}}\right) \text { and } \\
\nu\left(2^{n+p} f\left(\frac{x}{2^{n+p}}\right)-2^{p} f\left(\frac{x}{2^{p}}\right), t\right) \leq \nu^{\prime}\left(\varphi(x, 2 x, x), \frac{t}{\sum_{j=p}^{n+p-1} 2^{j}|\alpha|^{j+1}}\right) .
\end{array}\right\}
$$

Since $0<|\alpha|<\frac{1}{2}$, we have $\sum_{j=0}^{\infty}(2|\alpha|)^{j+1}<\infty$. This shows that $\left(2^{n} f\left(\frac{x}{2^{n}}\right)\right)$ is a Cauchy sequence in an intuitionistic fuzzy Banach space $(Y, \mu, \nu)$ and so it converges to some point 
$T(x) \in Y$. Thus, we define a mapping $T: X \rightarrow Y$ such that

$$
T(x)=(\mu, v)-\lim _{n \rightarrow \infty} 2^{n} f\left(\frac{x}{2^{n}}\right)
$$

Hence, for all $x \in X$ and $t>0$, we have

$$
\mu\left(2^{n} f\left(\frac{x}{2^{n}}\right)-T(x), t\right)=1 \quad \text { and } \quad v\left(2^{n} f\left(\frac{x}{2^{n}}\right)-T(x), t\right)=0
$$

Moreover, if we put $p=0$ in (4.6), we get

$$
\begin{aligned}
& \mu\left(2^{n} f\left(\frac{x}{2^{n}}\right)-f(x), t\right) \geq \mu^{\prime}\left(\varphi(x, 2 x, x), \frac{t}{\sum_{j=0}^{n-1} 2^{j}|\alpha|^{j+1}}\right) \text { and } \\
& v\left(2^{n} f\left(\frac{x}{2^{n}}\right)-f(x), t\right) \leq v^{\prime}\left(\varphi(x, 2 x, x), \frac{t}{\sum_{j=0}^{n-1} 2^{j}|\alpha|^{j+1}}\right),
\end{aligned}
$$

for all $x \in X, t>0$ and $n>0$. Therefore,

$$
\begin{aligned}
\mu(T(x)-f(x), t) & \geq \mu\left(T(x)-2^{n} f\left(\frac{x}{2^{n}}\right), t / 2\right) * \mu\left(2^{n} f\left(\frac{x}{2^{n}}\right)-f(x), t / 2\right) \\
& \geq \mu^{\prime}\left(\varphi(x, 2 x, x), \frac{t}{\sum_{j=0}^{n-1}(2|\alpha|)^{j+1}}\right)
\end{aligned}
$$

and

$$
\begin{aligned}
v(T(x)-f(x), t) & \leq v\left(T(x)-2^{n} f\left(\frac{x}{2^{n}}\right), t / 2\right) \diamond v\left(2^{n} f\left(\frac{x}{2^{n}}\right)-f(x), t / 2\right) \\
& \leq v^{\prime}\left(\varphi(x, 2 x, x), \frac{t}{\sum_{j=0}^{n-1}(2|\alpha|)^{j+1}}\right),
\end{aligned}
$$

for all $x \in X, t>0$ and $n>0$. Letting $n \rightarrow \infty$ in the above inequalities, we obtain

$$
\begin{aligned}
& \mu(T(x)-f(x), t) \geq \mu^{\prime}\left(\varphi(x, 2 x, x), \frac{(1-2|\alpha|) t}{|\alpha|}\right) \quad \text { and } \\
& \nu(T(x)-f(x), t) \leq v^{\prime}\left(\varphi(x, 2 x, x), \frac{(1-2|\alpha|) t}{|\alpha|}\right) .
\end{aligned}
$$

Hence, $T$ satisfies (4.3). Let $x, y, z \in X$. Then

$$
\begin{aligned}
& \mu\left(2 T\left(\frac{x+y+z}{2}\right)-T(x)-T(y)-T(z), t\right) \\
& \geq \mu\left(2 T\left(\frac{x+y+z}{2}\right)-2^{n+1} f\left(\frac{x+y+z}{2^{n+1}}\right), \frac{t}{5}\right) * \mu\left(2^{n} f\left(\frac{x}{2^{n}}\right)-T(x), \frac{t}{5}\right) \\
& \quad * \mu\left(2^{n} f\left(\frac{y}{2^{n}}\right)-T(y), \frac{t}{5}\right) * \mu\left(2^{n} f\left(\frac{z}{2^{n}}\right)-T(z), \frac{t}{5}\right) \\
& \quad * \mu\left(2^{n+1} f\left(\frac{x+y+z}{2^{n+1}}\right)-2^{n} f\left(\frac{x}{2^{n}}\right)-2^{n} f\left(\frac{y}{2^{n}}\right)-2^{n} f\left(\frac{z}{2^{n}}\right), \frac{t}{5}\right)
\end{aligned}
$$


and by using (4.2)

$$
\begin{gathered}
\mu\left(2^{n+1} f\left(\frac{x+y+z}{2^{n+1}}\right)-2^{n} f\left(\frac{x}{2^{n}}\right)-2^{n} f\left(\frac{y}{2^{n}}\right)-2^{n} f\left(\frac{z}{2^{n}}\right), t\right) \\
\geq \mu^{\prime}\left(\varphi\left(\frac{x}{2^{n}}, \frac{y}{2^{n}}, \frac{z}{2^{n}}\right), \frac{t}{2^{n}}\right) \geq \mu^{\prime}\left(\varphi(x, y, z), \frac{t}{2^{n}|\alpha|^{n}}\right) .
\end{gathered}
$$

Letting $n \rightarrow \infty$ in (4.7) and (4.8), we get

$$
\mu\left(2 T\left(\frac{x+y+z}{2}\right)-T(x)-T(y)-T(z), t\right)=1 .
$$

Similarly, we obtain

$$
v\left(2 T\left(\frac{x+y+z}{2}\right)-T(x)-T(y)-T(z), t\right)=0,
$$

for all $x, y, z \in X$ and $t>0$. This means that $T$ satisfies (1.1) and so it is additive. To prove the uniqueness of $T$, assume that $S$ be another additive mapping from $X$ into $Y$, which satisfies (4.3). For $x \in X$, clearly $T(x)=2^{n} T\left(\frac{x}{2^{n}}\right)$ and $S(x)=2^{n} S\left(\frac{x}{2^{n}}\right)$ for all $n$. It follows from (4.3) that

$$
\begin{aligned}
\mu(T(x)-S(x), t) & =\mu\left(2^{n} T\left(\frac{x}{2^{n}}\right)-2^{n} S\left(\frac{x}{2^{n}}\right), t\right) \\
& \geq \mu\left(2^{n} T\left(\frac{x}{2^{n}}\right)-2^{n} f\left(\frac{x}{2^{n}}\right), \frac{t}{2}\right) * \mu\left(2^{n} f\left(\frac{x}{2^{n}}\right)-2^{n} S\left(\frac{x}{2^{n}}\right), \frac{t}{2}\right) \\
& \geq \mu^{\prime}\left(\varphi\left(\frac{x}{2^{n}}, \frac{2 x}{2^{n}}, \frac{x}{2^{n}}\right), \frac{(1-2|\alpha|) t}{2^{n+1}|\alpha|}\right) \\
& \geq \mu^{\prime}\left(\varphi(x, 2 x, x), \frac{(1-2|\alpha|) t}{2^{n+1}|\alpha|^{n+1}}\right)
\end{aligned}
$$

and similarly

$$
v(T(x)-S(x), t) \leq v^{\prime}\left(\varphi(x, 2 x, x), \frac{(1-2|\alpha|) t}{2^{n+1}|\alpha|^{n+1}}\right) .
$$

We see that the right-hand side of (4.9) and (4.10) tending to 1 and 0 , respectively, as $n \rightarrow \infty$. Therefore, $\mu(T(x)-S(x), t)=1$ and $\nu(T(x)-S(x), t)=0$ for all $x \in X$ and $t>0$. Hence, $T(x)=S(x)$.

Corollary 4.2 Let $X$ be a normed linear space and $\left(\mathbb{R}, \mu^{\prime}, v^{\prime}\right)$ be an intuitionistic fuzzy Banach space. Let $\theta$ be a positive real number and $r$ is a real number with $0<r<1$. If a mapping $f: X \rightarrow Y$ satisfies the conditions,

$$
\begin{aligned}
& \mu\left(2 f\left(\frac{x+y+z}{2}\right)-f(x)-f(y)-f(z), t\right) \geq \mu^{\prime}\left(\theta\left(\|x\|^{r}+\|y\|^{r}+\|z\|^{r}\right), t\right) \quad \text { and } \\
& \nu\left(2 f\left(\frac{x+y+z}{2}\right)-f(x)-f(y)-f(z), t\right) \leq v^{\prime}\left(\theta\left(\|x\|^{r}+\|y\|^{r}+\|z\|^{r}\right), t\right),
\end{aligned}
$$


for all $x, y, z \in X$ and $t>0$, then there exists a unique additive mapping $T: X \rightarrow Y$ such that

$$
\mu(f(x)-T(x), t) \geq \mu^{\prime}\left(\theta\|x\|^{r}, \frac{2 t}{2^{\alpha}+2}\right) \quad \text { and } \quad v(f(x)-T(x), t) \leq v^{\prime}\left(\theta\|x\|^{r}, \frac{2 t}{2^{\alpha}+2}\right),
$$

for all $x \in X$ and $t>0$.

Proof Taking $\varphi(x, y, z)=\theta\left(\|x\|^{r}+\|y\|^{r}+\|z\|^{r}\right)$ in Theorem 4.1, for all $x, y, z \in X$, and choosing $|\alpha|=1 / 4$, we get the desired result.

Theorem 4.3 Let $X$ be a linear space and $\left(Z, \mu^{\prime}, v^{\prime}\right)$ be an IFN-space. Suppose that $\varphi$ : $X \times X \times X \rightarrow Z$ is a function such that for some real number $\alpha$ with $0<|\alpha|<2$

$$
\begin{aligned}
& \mu^{\prime}(\varphi(2 x, 2 y, 2 z), t) \geq \mu^{\prime}(|\alpha| \varphi(x, y, z), t) \quad \text { and } \\
& \nu^{\prime}(\varphi(2 x, 2 y, 2 z), t) \leq v^{\prime}(|\alpha| \varphi(x, y, z), t),
\end{aligned}
$$

for all $x, y, z \in X$ and $t>0$. Let $(Y, \mu, v)$ an intuitionistic fuzzy Banach space and a map $f: X \rightarrow Y$ satisfies (4.2). Then there exists a unique additive mapping $T: X \rightarrow Y$ such that

$$
\begin{aligned}
& \mu(f(x)-T(x), t) \geq \mu^{\prime}(\varphi(x, 2 x, x),(2-|\alpha|) t) \quad \text { and } \\
& v(f(x)-T(x), t) \leq v^{\prime}(\varphi(x, 2 x, x),(2-|\alpha|) t),
\end{aligned}
$$

for all $x \in X$ and $t>0$.

Proof From (4.4), it is easy to see that

$$
\begin{aligned}
& \mu\left(\frac{f(2 x)}{2}-f(x), \frac{t}{2}\right) \geq \mu^{\prime}(\varphi(x, 2 x, x), t) \quad \text { and } \\
& \nu\left(\frac{f(2 x)}{2}-f(x), \frac{t}{2}\right) \leq v^{\prime}(\varphi(x, 2 x, x), t),
\end{aligned}
$$

for all $x \in X$ and $t>0$. Replacing $x$ by $2^{n} x$, we get

$$
\begin{aligned}
& \mu\left(\frac{f\left(2^{n+1} x\right)}{2^{n+1}}-\frac{f\left(2^{n} x\right)}{2^{n}}, \frac{t}{2^{n+1}}\right) \geq \mu^{\prime}\left(\varphi\left(2^{n} x, 2^{n+1} x, 2^{n} x\right), t\right) \geq \mu^{\prime}\left(\varphi(x, 2 x, x), \frac{t}{|\alpha|^{n}}\right) \text { and } \\
& \nu\left(\frac{f\left(2^{n+1} x\right)}{2^{n+1}}-\frac{f\left(2^{n} x\right)}{2^{n}}, \frac{t}{2^{n+1}}\right) \leq \nu^{\prime}\left(\varphi\left(2^{n} x, 2^{n+1} x, 2^{n} x\right), t\right) \leq \nu^{\prime}\left(\varphi(x, 2 x, x), \frac{t}{|\alpha|^{n}}\right)
\end{aligned}
$$

It follows that, for all $x \in X$ and $t>0$, we have

$$
\begin{aligned}
& \mu\left(\frac{f\left(2^{n+1} x\right)}{2^{n+1}}-\frac{f\left(2^{n} x\right)}{2^{n}}, \frac{|\alpha|^{n} t}{2^{n+1}}\right) \geq \mu^{\prime}(\varphi(x, 2 x, x), t) \quad \text { and } \\
& \nu\left(\frac{f\left(2^{n+1} x\right)}{2^{n+1}}-\frac{f\left(2^{n} x\right)}{2^{n}}, \frac{|\alpha|^{n} t}{2^{n+1}}\right) \leq \nu^{\prime}(\varphi(x, 2 x, x), t) .
\end{aligned}
$$


Proceeding the same lines as in the proof of Theorem 4.1, we get

$$
\begin{aligned}
& \mu\left(\frac{f\left(2^{n} x\right)}{2^{n}}-f(x), \sum_{j=0}^{n-1} \frac{|\alpha|^{j} t}{2^{j+1}}\right) \geq \mu^{\prime}(\varphi(x, 2 x, x), t) \quad \text { and } \\
& \nu\left(\frac{f\left(2^{n} x\right)}{2^{n}}-f(x), \sum_{j=0}^{n-1} \frac{|\alpha|^{j} t}{2^{j+1}}\right) \leq \nu^{\prime}(\varphi(x, 2 x, x), t),
\end{aligned}
$$

for all $x \in X, t>0$ and $n>0$. Thus,

$$
\begin{aligned}
& \mu\left(\frac{f\left(2^{n} x\right)}{2^{n}}-f(x), t\right) \geq \mu^{\prime}\left(\varphi(x, 2 x, x), \frac{t}{\sum_{j=0}^{n-1} \frac{|\alpha|^{j}}{j^{j+1}}}\right) \geq \mu^{\prime}(\varphi(x, 2 x, x),(2-|\alpha|) t) \quad \text { and } \\
& \nu\left(\frac{f\left(2^{n} x\right)}{2^{n}}-f(x), t\right) \leq \nu^{\prime}\left(\varphi(x, 2 x, x), \frac{t}{\sum_{j=0}^{n-1} \frac{|\alpha|^{j}}{2^{j+1}}}\right) \leq \nu^{\prime}(\varphi(x, 2 x, x),(2-|\alpha|) t) .
\end{aligned}
$$

Rest of the proof can be done by the same way as in Theorem 4.1.

Corollary 4.4 Let $X$ be a normed linear space and $\left(\mathbb{R}, \mu^{\prime}, v^{\prime}\right)$ be an intuitionistic fuzzy Banach space. Let $\theta$ be a positive real number and $r$ is a real number with $0<r<\frac{1}{3}$. If a mapping $f: X \rightarrow Y$ satisfies the conditions

$$
\begin{aligned}
& \mu\left(2 f\left(\frac{x+y+z}{2}\right)-f(x)-f(y)-f(z), t\right) \geq \mu^{\prime}\left(\theta\left(\|x\|^{r}+\|y\|^{r}+\|z\|^{r}\right), t\right) \quad \text { and } \\
& \nu\left(2 f\left(\frac{x+y+z}{2}\right)-f(x)-f(y)-f(z), t\right) \leq v^{\prime}\left(\theta\left(\|x\|^{r}+\|y\|^{r}+\|z\|^{r}\right), t\right)
\end{aligned}
$$

for all $x, y, z \in X$ and $t>0$, then there exists a unique additive mapping $T: X \rightarrow Y$ such that

$$
\mu(f(x)-T(x), t) \geq \mu^{\prime}\left(\theta\|x\|^{r}, \frac{t}{2^{r}+2}\right) \quad \text { and } \quad v(f(x)-T(x), t) \leq v^{\prime}\left(\theta\|x\|^{r}, \frac{t}{2^{r}+2}\right),
$$

for all $x \in X$ and $t>0$.

Proof Taking $\varphi(x, y, z)=\theta\left(\|x\|^{r}+\|y\|^{r}+\|z\|^{r}\right)$ in Theorem 4.3, for all $x, y, z \in X$, and choosing $|\alpha|=1$, we get the desired result.

\section{Competing interests}

The authors declare that they have no competing interests.

Authors' contributions

Both the authors contributed equally and significantly in writing this paper. All authors read and approved the final manuscript.

\section{Acknowledgements}

The authors would like to thank the anonymous reviewers for their valuable comments.

Received: 26 May 2012 Accepted: 3 August 2012 Published: 8 August 2012 


\section{References}

1. Agarwal, RP, Xu, B, Zhang, W: Stability of functional equations in single variable. J. Math. Anal. Appl. 288, $852-869$ (2003)

2. Alotaibi, A, Mohiuddine, SA: On the stability of a cubic functional equation in random 2-normed spaces. Adv. Differ. Equ. 2012, 39 (2012)

3. Aoki, T: On the stability of the linear transformation in Banach spaces. J. Math. Soc. Jpn. 2, 64-66 (1950)

4. Diaz, JB, Margolis, B: A fixed point theorem of the alternative for contractions on generalized complete metric space. Bull. Am. Math. Soc. 126(74), 305-309 (1968)

5. Hyers, DH: On the stability of the linear functional equation. Proc. Natl. Acad. Sci. USA 27, $222-224$ (1941)

6. Hyers, DH, Isac, G, Rassias, TM: Stability of Functional Equations in Several Variables. Birkhäuser, Basel (1998)

7. Gajda, Z: On stability of additive mappings. Int. J. Math. Math. Sci. 14, 431-434 (1991)

8. Găvruta, P: A generalization of the Hyers-Ulam-Rassias stability of approximately additive mappings. J. Math. Anal. Appl. 184, 431-436 (1994)

9. Jung, SM: Hyers-Ulam-Rassias stability of Jensen's equation and its application. Proc. Am. Math. Soc. 126, 3137-3143 (1998)

10. Jung, SM: Hyers-Ulam stability of zeros of polynomials. Appl. Math. Lett. 24, 1322-1325 (2011)

11. Kenary, HA, Rezaei, H, Ghaffaripour, A, Talebzadeh, S, Park, C, Lee, JR: Fuzzy Hyers-Ulam stability of an additive functional equation. J. Inequal. Appl. 2011, 140 (2011)

12. Lee, Y-H, Jun, K-W: A generalization of the Hyers-Ulam-Rassias stability of Jensen's equation. J. Math. Anal. Appl. 238 305-315 (1999)

13. Miheț, D: The fixed point method for fuzzy stability of the Jensen functional equation. Fuzzy Sets Syst. 160 1663-1667 (2009)

14. Mohiuddine, SA, Alotaibi, A: Fuzzy stability of a cubic functional equation via fixed point technique. Adv. Differ. Equ. 2012, 48 (2012)

15. Mohiuddine, SA, Şevli, $\mathrm{H}$ : Stability of pexiderized quadratic functional equation in intuitionistic fuzzy normed space. J. Comput. Appl. Math. 235, 2137-2146 (2011)

16. Mohiuddine, SA, Cancan, M, Şevli, H: Intuitionistic fuzzy stability of a Jensen functional equation via fixed point technique. Math. Comput. Model. 54, 2403-2409 (2011)

17. Mohiuddine, SA: Stability of Jensen functional equation in intuitionistic fuzzy normed space. Chaos Solitons Fractals 42, 2989-2996 (2009)

18. Mohiuddine, SA, Danish Lohani, QM: On generalized statistical convergence in intuitionistic fuzzy normed space. Chaos Solitons Fractals 42, 1731-1737 (2009)

19. Mursaleen, M, Karakaya, $V$, Mohiuddine, SA: Schauder basis, separability, and approximation property in intuitionistic fuzzy normed space. Abstr. Appl. Anal. 2010, Article ID 131868 (2010)

20. Mursaleen, M, Mohiuddine, SA: Statistical convergence of double sequences in intuitionistic fuzzy normed spaces. Chaos Solitons Fractals 41, 2414-2421 (2009)

21. Mursaleen, M, Mohiuddine, SA: On stability of a cubic functional equation in intuitionistic fuzzy normed spaces. Chaos Solitons Fractals 42, 2997-3005 (2009)

22. Mursaleen, M, Mohiuddine, SA: On lacunary statistical convergence with respect to the intuitionistic fuzzy normed space. J. Comput. Appl. Math. 233(2), 142-149 (2009)

23. Mursaleen, M, Mohiuddine, SA: Nonlinear operators between intuitionistic fuzzy normed spaces and Fréchet differentiation. Chaos Solitons Fractals 42, 1010-1015 (2009)

24. Mursaleen, M, Mohiuddine, SA, Edely, OHH: On the ideal convergence of double sequences in intuitionistic fuzzy normed spaces. Comput. Math. Appl. 59, 603-611 (2010)

25. Radu, V: The fixed point alternative and the stability of functional equations. Fixed Point Theory 4(1), 91-96 (2003)

26. Rassias, TM: On the stability of the linear mapping in Banach spaces. Proc. Am. Math. Soc. 72, 297-300 (1978)

27. Rassias, TM: On the stability of functional equations and a problem of Ulam. Acta Appl. Math. 62, 123-130 (2000)

28. Saadati, R, Park, JH: On the intuitionistic fuzzy topological spaces. Chaos Solitons Fractals 27, 331-344 (2006)

29. Wang, Z, Rassias, TM: Intuitionistic fuzzy stability of functional equations associated with inner product spaces. Abstr. Appl. Anal. 2011, Article ID 456182 (2011)

30. Ulam, SM: A Collection of the Mathematical Problems. Interscience, New York (1960)

31. Xu, TZ, Rassias, JM, Xu, WX: Intuitionistic fuzzy stability of a general mixed additive-cubic equation. J. Math. Phys. 51, $063519(2010)$

32. Xu, TZ, Rassias, JM, Xu, WX: Stability of a general mixed additive-cubic functional equation in non-Archimedean fuzzy normed spaces. J. Math. Phys. 51, 093508 (2010)

33. Xu, TZ, Rassias, JM: A Fixed point approach to the stability of an AQ-functional equation on beta-Banach modules. Fixed Point Theory Appl. 2012, 32 (2012)

34. Yilmaz, Y: On some basic properties of differentiation in intuitionistic fuzzy normed spaces. Math. Comput. Model. $\mathbf{5 2}$, 448-458 (2010)

doi:10.1186/1687-1847-2012-141

Cite this article as: Mohiuddine and Alghamdi: Stability of functional equation obtained through a fixed-point

alternative in intuitionistic fuzzy normed spaces. Advances in Difference Equations 2012 2012:141. 